\title{
M-HEALTH AN EMERGING TREND AN EMPIRICAL STUDY
}

\author{
Muhammed Fuzail Zubair, Hajrah Jahan, Dr. Sophia Rahaman and \\ Dr. Roma Raina
}

\author{
School of Engineering and IT, Manipal University, Dubai, UAE \\ fuzailmuhammed@gmail.com \\ hajrah.jahan@yahoo.com \\ sophia@manipaldubai.com \\ roma.raina@manipaldubai.com
}

\begin{abstract}
The advent and advancement in technology specific to medical field has seen a migration of its work across the globe, adapting higher and newer levels of m-health. Technology has been successful in transforming the way traditional monitoring and alert system work to a modern approach wherein minimizing the need for physical monitoring. Today, the field of healthcare use varied monitoring systems to monitor the health of patients using ubiquitous and nonubiquitous devices. These are sensor based devices that can read vital signs of patients and send the data to the required personnel's using mobile networks. This paper understands and analyses how the monitoring and alert system works specific to m-health. m-health including wearable and non-wearable devices read various vital signs and have the ability to monitor health real-time and transfer the information collected using mobile network. m-health has become an useful tool for elderly in this fast paced world where almost all the family members are working or studying to keep track and maintain optimal health status. m-health alert system involves the patient, the caretaker and medical service provider wherein the patient wears the device and vital signs recorded are transferred the medical service provider who then analyses the data collected and required changes in the medication are implemented. This paper proposes a medical alert system that enlightens the capabilities of $m$-health making health monitoring easy and reliable. It contains a three-level severity check and raises an alarm to the caretaker, the physician or the ambulatory service provider.
\end{abstract}

\section{KEYWORDS}

e-health; m-health; Wearable Wireless Sensor based Area Network; Data Mining.

\section{INTRODUCTION}

The advent of mobile technology and its services are quickly emerging as the new frontier in transforming the public and private sectors of the society, trying to make it even more accessible and customer-centric by extending the benefits of remote delivery [1]. Their main objective is to provide a $24 \times 7$ service across the globe via mobile networks. Particularly in the field of medicine David C. Wyld et al. (Eds) : CCSEA, CLOUD, DKMP, SEA, SIPRO - 2016 pp. 167-174, 2016. @ CS \& IT-CSCP 2016

DOI : $10.5121 / \mathrm{csit} .2016 .60215$ 
the approach has elevated healthcare practice to a new level that imbibes computing power backed by the electronic communication technology [2].This approach spans across various services like, Electronic health records, Computerized physician order entry, e-prescribing, Clinical Decision Support, Telemedicine, Consumer health informatics, Health knowledge management, Medical research using Grids [3] \& Healthcare Information Systems.

e-health is categorized as ubiquitous where the monitored health resides on a particular location and is communicated to the interested personal via direct connection to the server. For example, World Health Organization's consumer health information service. On the other hand is nonubiquitous where the communication uses wireless technology in communicating the information to the user, e.g. $\mathrm{CH}$ Telemetry System by ApexPro. Latest advancements are related to another term called $\mathrm{m}$-Health which is monitoring and communicating medical conditions using mobile technology $[4,5]$. The fast pace adaption to $\mathrm{m}$-health is due to the major shift in health care needs towards more scalable and affordable solutions. This is restructuring the healthcare systems towards proactive management of wellness rather than illness, which is focused at prevention and early detection of disease [6].

\section{MOTIVATION AND OBJECTIVE}

The classification of m-health has routed to a path of non-wearable and wearable devices. The non-wearable technology in m-health constitutes of applications to sense the heartbeat, blood pressure, oxygen saturation, UV exposure, pedometer etc. and is widely used by the leading mobile manufacturers like Samsung \& Apple. These technologies require a head on to start their process and provide an output. On the contrary, wearable technologies focus is to provide a real time health monitoring service to maintain optimal health status. When integrated into a telemedical system they raise a medical alert in life threatening scenarios. In addition, patients also benefit in terms of continuous long term monitoring as a part of a diagnostic procedure to achieve optimal maintenance of a chronic condition, or supervision during recovery from an acute event or surgical procedure.

Wearable health monitoring systems [7] integrated into a telemedicine system is a novel information technology approach that will be able to support early detection of abnormal conditions and prevention of its serious consequences [8]. The wearable devices shelter numerous sensors required for monitoring health conditions like, pulse, blood pressure, temperature and others [9]. These devices worn or carried by the patient, monitor the condition on a regular basis and provide real time information to the receiving end of the communicating device, particularly mobile phones in this context, via applications, IM, links etc. Keeping in view that health monitoring has the potential to improve the quality of health services \& ensuring that those who need urgent care get it sooner, this research aims to study and understand the varied dimensions of such systems $[10,11,12]$.

\section{METHODOLOGY}

Recent technology advances in integration and miniaturization of physical sensors, embedded microcontrollers and radio interfaces on a single chip; wireless networking; and micro-fabrication have enabled a new generation of wireless sensor networks suitable for such applications by the use of Wearable Wireless Sensor based Area Network (WWSAN) [13,14]. The WWSAN consisting of inexpensive, lightweight, and miniature sensors carry long-term, unobtrusive, 
ambulatory health monitoring with instantaneous feedback to the user about the current health status and real-time or near real-time update of the user's medical records. Intelligent heart monitors warning users about impeding medical conditions[15],Accelerometer-based system monitoring physical activity [16] are a few to name.

The wearable device can include a number of physiological sensors and photoplethysmographic biosensors [17] depending on the end-user application. An extensive set of physiological sensors may include an (electrocardiogram) sensor for monitoring heart activity, an EMG (electromyography) sensor for monitoring muscle activity, a blood pressure sensor, a temperature sensor, an EEG (electroencephalography) sensor for monitoring electrical activity of the brain etc.

The WWSAN when integrated into a broader telemedical system with patients' medical records promises a revolution in medical research with the use of data mining on the gathered information. This enables the researchers to explore the synergy between varied parameters of the collated data giving scope to perform quantitative analysis of various conditions and patterns that will built be with time. The Sensors on the wearable device can be integrated into various objects such as garments, wrist bands, socks, shoes etc. Some of the sensors that are invasive in nature are implanted on to the body. The sensor plays a significant role by collecting various data easily through signals on a chip that is ingested or worn by the patient body and helps in storing the various changes that occur in an organized form on a device the sensor is connected to and provide an alert to immediate caretakers and other emergency contacts based on the severity of variation as depicted in figure.

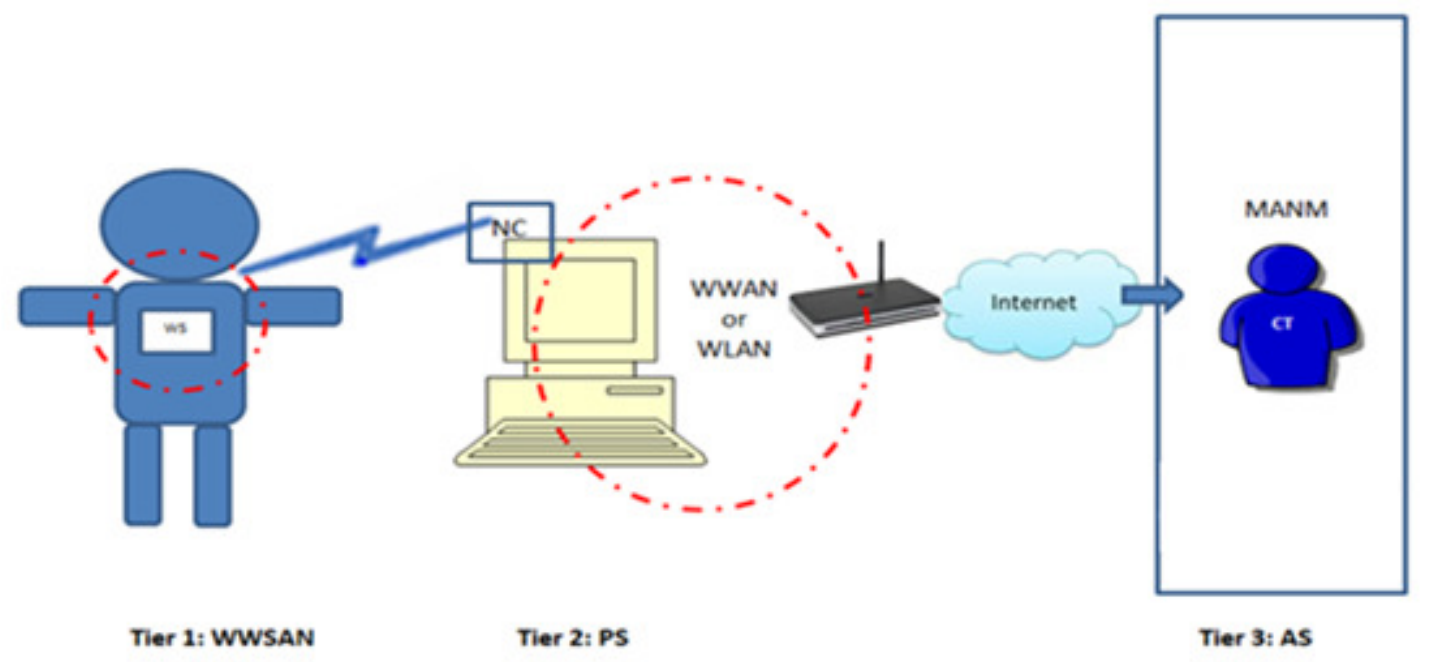

Figure 1: Three Tier Medical

As depicted in Figure 1, the first tier is patient wearing the sensor (WS), via the sensor nodes, it senses and processes the varied physiological signals. The WWSAN capture these signals and via Network Configuration (NC) transmit them to tier 2 that hosts the personal server (PS). The PS provides transparent interfaces to the WS; the device then uses the WWAN or WLAN and via the internet raises a medical alert notification message (MANM). Finally PS communicates the medical condition to the $3^{\text {rd }}$ tier, Alert System(AS) which raises a MANM based on severity of the patient's condition either to the care taker (CT) or an emergency contact apart from updating 
the patient medical record. This technology can improve the quality of life of elderly and dependent people who require medical attention frequently and immediately [18].

\section{DISCUSSION}

The proposed methodology in the previous section is further explained with the use of case studies. Two case studies are chosen to illustrate working of WS in a health monitoring system provided to the target group over the pilot period of the experiment.

First case study is of CHRISTUS Health System [19] by St. Michael Hospital, Texarkana. They used CHRISTUS Health System to monitor the health of high-risk patients diagnosed with chronic illness such as Congestive Heart Failure, Hypertension, and Diabetes. This health system consists of Remote Patient Management System, Remote Patient Monitoring System that includes Android tablet and Bluetooth paired personal health devices: weight scale, blood pressure monitor, pulse oximeter. In this health system, patient protocols and care plans are easily customized for each patient and an user interface is provided for the patients to use. At the end of the program, results showed a $90 \%$ reduction in overall cost of care, $65 \%$ reduction in hospital readmissions, $95 \%$ patient adoption and patient satisfaction.

Second case study is of Ambio Health Remote Patient Monitoring conducted by Enjoy Life! [20] Health Consulting. Diabetic patients were invited to participate in a pilot program in which they were given blood glucose meter, blood glucose strips and supporting equipment to send their blood glucose reading wirelessly. Blood pressure monitor was also provided based on the requirement. A patient who volunteered for this program had a history type 2 diabetes and high blood pressure. The patient never used to check blood pressure and would forget the blood sugar levels or doctor found the blood sugar levels provided by him were hundred percent correct. After using this system, his health care provider found out that all the numbers were high based on the data collected and prompt action was taken with change in medication in consultation with the patient's physician.

The underlying application of data mining enables the learners to digest large amounts of data by leveraging sophisticated techniques in data analysis, restructuring and organization [21]. In a datamining system the data is preprocessed and suitable mining technique is applied on the relevant data that are generally descriptive and predictive in nature and generate patterns as knowledge to the user. Some of the commonly used techniques for predictive analysis specific to healthcare are as discussed in brief :

Clustering - This approach is the stratification of objects into different groups, precisely partitioning the data set into clusters or subsets. The subset ideally shares certain common straits. This technique is suitable for statistical data analysis used in fields like machine learning, pattern recognition, image analysis and bioinformatics.[22]

Classification - This approach is known for its categorization of data for efficient and effective use. Proper classification of patient health records aid in verification, diagnosis and in-depth data processing. Classification tree and rules are commonly used to obtain simple models that are employed in clinics. Recent examples of such models include classification of cancer using gene expression data, classification of tumors of the tongue to name a few. [23,24] 
Association rule mining - This approach enables the users in finding interesting patterns and trends in data. Association rules identify collections of data attributes that are statistically related in the underlying data. This approach brings out the precautionary measures that can be adapted for better health management. [25, 26, 27]

Sequential mining - This technique in specific is focused in finding inter-transaction patterns, to detect the presence of a set of events in a time ordered sequence of transactions. Here an order exists between the occurring events with a possibility of an event re-occurring in the same order. This functionality makes it aptly suitable to the healthcare sector in predicting future health risks and educating in better health management.[28, 29].

\section{PROPOSED MODEL}

An analysis of the cases discussed in the above section brings to light the importance of remote health monitoring system. It emphasizes on the availability, ease of use, readiness and significance of such systems. The proposed model depicted in figure 2 , illustrates the process flow for these systems.

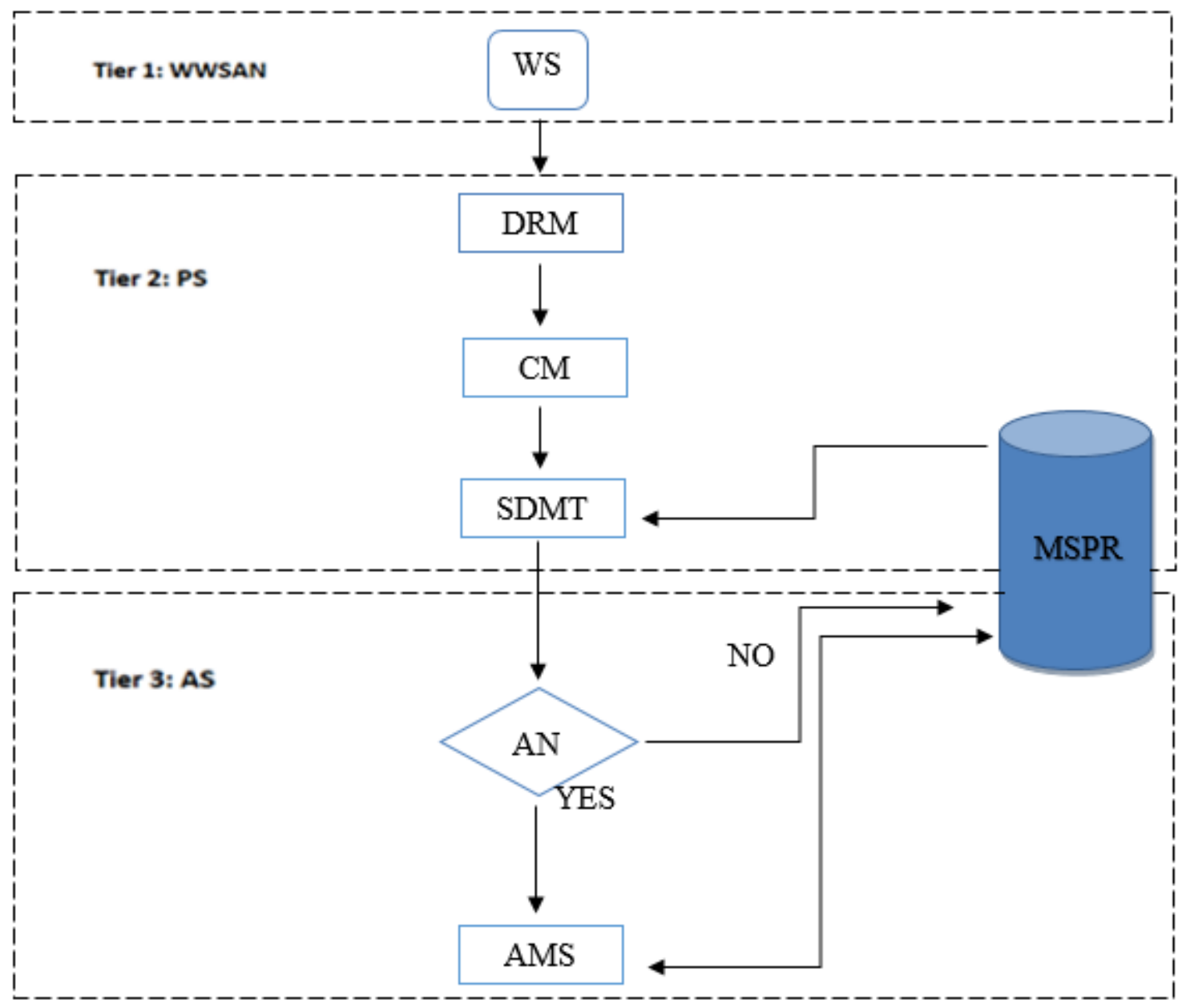

Figure 2: Process flow of the proposed model 
The proposed model states the wearable sensor (WS) senses the required vital readings and transmits them to the Data Receiver Module (DRM) in the second tier: PS. The DRM processes the data as per the measurement of the system and passes it to the Compilation Module (CM). The compiled data in now further analysed by the application of Suitable Data Mining Techniques (SDMT). The analysis results are passed to the Alert Notification Module (ANM) in tier 3: AS. The AVM raises an alert based on the severity of the patient's condition to the notification receiver (emergency contact). The Ambulatory Service (AMS) and the Medical Service Provider (MSP) get the access to the PRMH along with the Condition Prevalent Data (CPD).

\section{CONCLUSION AND FURTHER RESEARCH}

This paper demonstrates the use of WWSAN as a key infrastructure enabling unobtrusive, continual, ambulatory health monitoring. This emerging technology offers wide range of benefits to patients, medical personnel, and the society through continuous monitoring in the ambulatory setting, early detection of abnormal conditions, supervised rehabilitation, and potential knowledge discovery through data mining from the gathered information.

The increasing development of mobile health care system yields the largest growth among mobile users. Mobile healthcare alert system that delivers the proper timing and emergency case alerts is considered advantageous related to power consuming, portability and flexibility as the mobility devices enhance the computation based on the ubiquitous nature. In addition, further studies of varied medical conditions in clinical and ambulatory settings are essential to determine specific limitations and possible new and applications of this emerging technology.

\section{REFERENCES}

[1] Park. S, (2003) "Enhancing The Quality Of Life Through Wearable Technology", Engineering In Medicine And Biolog Magazine, IEEE, Volume:22 Issue: 3.

[2] Della Mea \& Vincenzo (2001) "What Is E-Health (2): The Death Of Telemedicine?", Journal Of Medical Internet Research 3.

[3] Jochen Fingberg \& Marit Hansen Et Al., (2006)"'Integrating Data Custodians In Ehealth Grids Security And Privacy Aspects", NEC Lab Report.

[4] Adibi \& Sasan, (2015) "Mobile Health: A Technology Road Map. Springer”, Ed. February 19, Isbn 978-3-319-12817-7.

[5] K. Kiran Reddy, P.Lalith Samanth Reddy \& Dr.P.Bhaskara Reddy, (2014) "Study On Mobile Healthcare System", IJARCSSE, Volume 4, Issue 3, March. Available At: Www.Ijarcsse.Com

[6] Aleksandar Milenković, Chris Otto \& Emil Jovanov, (2006) "Wireless Sensor Networks For Personal Health Monitoring: Issues And An Implementation".

[7] Rutherford \& J.J, (2010) "Wearable Technology", Engineering In Medicine And Biolog Magazine, IEEE, Volume:29 Issue: 3.

[8] Istepanian RSH, Jovanov E \& Zhang YT, (2004) Guest Editorial Introduction To The Special Section On M-Health: "Beyond Seamless Mobility And Global Wireless Health-Care Connectivity" IEEE Transactions On Information Technology In Biomedicine, 8(4):405-414,Pubmed Abstract.

[9] Dr. Rajender Thusu, (2011) "Medical Sensors Facilitate Health Monitoring",Frost \& Sullivansensors, 1st April. Available At:

Http://Www.Sensorsmag.Com/Specialty-Markets/Medical/Sensors-Facilitate-Health-Monitoring8365

[10] Bonato. P , (2010) "Wearable Sensors And Systems", Engineering In Medicine And Biolog Magazine, IEEE, Volume:29 Issue: 3. 
[11] Ching Hsu, Member \& IACSIT, (2013) "Wireless Context-Aware Healthcare System Based On Sensor", Web 2.0 I-, IJI, Management And Technology, Vol. 4, No. 4, August.

[12] Jessica Gomez, (2014) "25 Most Interesting Medical MEMS And Sensor Projects”, Rogue Valley Microdevices. Available At: Http://Www.Slideshare.Net/Mikepinelisphd/140804-25-Most-Interesting-Medical-Mems-Sensors

[13] E. Jovanov, A. Milenkovic, C. Otto \& P. C. De Groen, (2005) "A Wireless Body Area Network Of Intelligent Motion Sensors For Computer Assisted Physical Rehabilitation," Journal Of Milenkovic Et. Al. Neuroengineering And Rehabilitation, 2:6, 1st March. Available At: [Http://Www.Jneuroengrehab.Com/Content/2/1/6]

[14] Jeonggil Ko, Chenyang Lu, Mani B. Srivastava, John A. Stankovic, Andreas Terzis \& Matt Welsh, (2010) "Wireless Sensor Networks For Healthcare", Proceedings Of The IEEE, Vol. 98, No. 11, November.

[15] J. Welch, F. Guilak \& S.D. Baker, (2004) "A Wireless ECG Smart Sensor For Broad Application In Life Threatening Event Detection”, In Proceedings Of The 26th Annual International Conference Of The IEEE Engineering In Medicine And Biology Society, (San Francisco, CA, September), Pp. $3447-$ 3449.

[16] M.J. Mathie \& B.G. Celler, (2001) “A System For Monitoring Posture And Physical Activity Using Accelerometers", In Proceedings Of The 23rd Annual International Conference Of The IEEE Engineering In Medicine And Biology Society, 2001, Pp. 3654- 3657.

[17] Asada, H.H, (2003) "Mobile Monitoring With Wearable Photoplethysmographic Biosensors", Engineering In Medicine And Biology Magazine, IEEE, Volume:22 Issue: 3.

[18] V.Barath \& P.Anithapriya, (2013) "A Survey On Healthcare For Aged People Based On Sensor Network", IJIRCCE, Volume:1 Issue:8, October.

[19] Christus Health System, St. Michael Hospital, (2014) "Remote Patient Monitoring for Care Transition Intervention Program, utilizing Remote Patient Monitoring System (RPMS) from Vivify Health".

[20] Leading Age Cast - Centre for aging services technologies, (2013) "Telehealth And Remote Patient Monitoring (RPM)".

[21] Guangming Li, (2013) "Research On The Medical Sub- Health Status Prediction And Future Selection”, IJACT Vol 5, No. 2, Pp 226 232.

[22] Khaled Hammoudal \& Mohammed Kamel, (2005) "Data Mining In E- Learning”, Pattern Analysis And Machine Intelligence (PAMI) Research Group, University Of Waterloo, Canada, 19 July.

[23] Chan Sheung Wai \& Clement Leung, (2013) "Clinical Decision Suport Systems For Acute Leukemia Classification In Co-Developments”, JDCTA, Vol 7, No. 1, Pp232 239.

[24] Fabricio Voznika \& Leonardo Viana, (2007) "Datamining Classification".

[25] C.S.Kanimozhi Selvi \& A.Tamilarasi, (2009) “An Automated Association Rule Mining Technique With Cumulative Support Thresholds”, Int. J. Open Problems In Compt. Math, Vol. 2, No. 3, September, ISSN 1998-6262; Copyright (C) ICSRS Publication. Available At: Www.I-Csrs.Org

[26] Gary S. Firestein, Ralph C. Budd, Sherine E. Gabriel, Iain Mclnnes \& James R O’Dell, (2000) "Kelly's Textbook Of Rheumatology", WB Saunders Co., Edited By Shuan Ruddy.

[27] Lippincott Williams \& Wilkens, (2003) "Clinical Primer Rheumatology", Edited By William Koopman Et, Al, Association.

[28] Ji`r'I Klema, Lenka Nov' Akov' A, Filip Karel, Olga ' $\mathrm{St}^{\vee} \mathrm{Ep}^{`}$ Ankov ' A \& Filip ' Zelezn ` Y, (2008) "Sequential Data Mining: A Comparative Case Study In Development Of Atherosclerosis Risk Factors", IEEE Transactions On Systems, Man, And Cybernetics-Part C: Applications And Reviews, Vol. 38, No. 1, January.

[29] Sophia Banu Rahaman \& Prof. M. Shashi, (2011) "Sequential Mining Equips E-Health With Knowledge For Managing Diabetes”, IJIPM, Vol. 2, No. 3, Pp. 74 85. 


\section{AUTHORS}

\section{Muhammed Fuzail Zubair}

Author is currently pursuing undergraduate degree in B.Sc Information System and Management with Networks as his specialization. His interest lies in Internet of Things (IOT), Cloud Technologies and Network Technologies. Excellent programmer with knowledge in networks and IT hardware, keen in taking up interesting and challenging projects.

\section{Hajrah Jahan}

Author is currently pursuing undergraduate degree in B.Sc Information System and Management with Software as her specialization. Her interest lies in developing applications using various programming languages. An ambitious programmer, keen in taking up interesting projects and an aspiring writer.

\section{Dr. Sophia Rahaman}

Author currently works as Professor in Manipal University,Dubai, imparts knowledge to students of Information Technology and Computer Science Engineering. Her area of interest lies in Databases, Data Mining and Digital World. Her area of expertise lies in Approaches and Technologies of Sequential Mining, E Health and E Learning. Author has published several research papers in her area of expertise

\section{Dr. Roma Raina}

Author currently works as Professor in Manipal University, Dubai, imparts knowledge to students of Engineering. Her area of interest lies in Controls \& Instrumentation. Her area of expertise lies in Radical Power Distribution, Controls \& Intstrumentation, Digital Electronics and Electrical Machines. Author has published several research papers in her area of expertise.
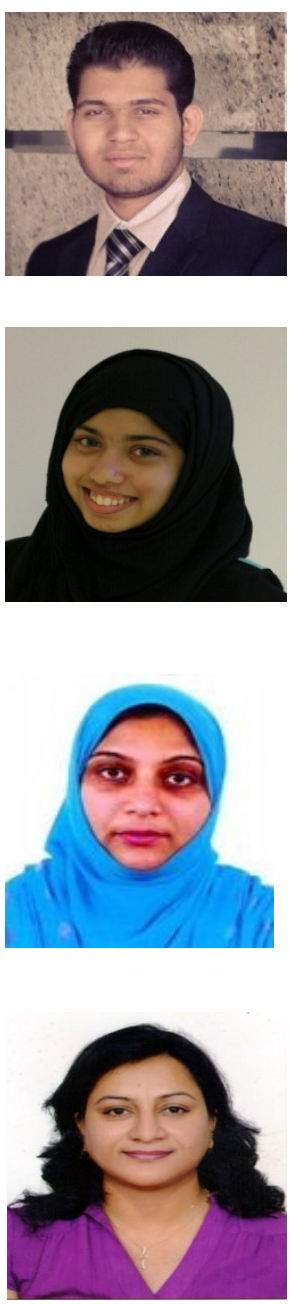